\title{
FDFD and FDTD analysis of 2-Dimensional lossy photonic crystals
}

\author{
Amin Gul Hanif ${ }^{a}$, Toru Uno, and Takuji Arima ${ }^{\text {b) }}$ \\ Electronic and Information Department of Graduate School of Engineering, \\ Tokyo University of Agriculture and Technology 2-24-16 Naka-cho, Koganei-shi, \\ Tokyo, Japan 184-0012 \\ a) 50009834203@ @t.tuat.ac.jp \\ b) t-arima@cc.tuat.ac.jp
}

Abstract: In this paper, a new FDFD algorithm for calculating a band gap diagram of 2-Dimensional (2D) lossy photonic crystals (PC) is developed. It is also shown that the FDTD method can not be successfully applied to the lossy PCs.

Keywords: FDFD, photonic crystal, lossy material, band diagram, FDTD

Classification: Electromagnetic theory

\section{References}

[1] F. Yang, Y. Rahmat-Samii, S. C. Cripps, P. Aaen, and J. A. Pla, "Electromagnetic Band Gap Structures in Antenna Engineering," Cambridge Univ. Press, 2008.

[2] Special Issue on Metamaterial Structures Phenomena and Applications, IEEE Trans. Microw. Tech., vol. 52, no. 2, Feb. 2004.

[3] K. Sakoda, Optical properties of photonic crystals, Springer-Verlag, Berlin, 2005.

[4] Special Issues on Electromagnetic Application of photonic bandgap structure, Progress in electromagnetics, PIER, vol. 41, 2003.

[5] J. D. Joannopoulos, R. D. Meade, and J. N. Winn, Photonic Crystal: Molding the Flow of Light, Princeton University Press, Princeton, NJ, 1995.

[6] K. S. Kunz and R. J. Luebbers, "The Finite Difference Time Domain Method for Electromagnetics," Boca Raton: CRC Press, 1993.

[7] T. Uno, "Finite Difference Time Domain Method for Electromagnetic Field and Antenna Analysis," Corona publishing Co. LTD. 1998. (in Japanese)

[8] S. Guo, F. Wu, S. Albin, and R. Rogowski, "Photonic band gap analysis using finite-difference frequency-domain method," Opt. Express, vol. 12, no. 8, pp. 1741-1746, April 2004.

[9] A. G. Hanif, Y. Kushiyama, T. Uno, and T. Arima, "FDFD and FDTD Methods for Band Diagram Analysis of 2- Dimensional Periodic Structure," IEICE Trans. Commun., vol. E93-B, no. 10, pp. 2670-2672, Oct. 2010. 


\section{Introduction}

There has been a great interest in development of new artificial structures referred as metamaterials in the fields of optics, microwave and antenna engineering $[1,2,3,4]$. Many applications have also been proposed for photonic devices, microwave components and antennas. The band diagram is one of the most important information for developing metamaterials including photonic crystals (PCs). Therefore, a variety of numerical methods have been utilized to calculate it. The most commonly used methods are the plane-wave expansion (PWE) method [5] and the finite-difference time-domain (FDTD) method $[6,7]$. In PWE method, the frequencies and its corresponding fields (eigenvalues and eigenvectors) can be found via an iterative process, but its resultant matrix is dense and large. For this reason, its computation resources becomes heavy for large problems. Moreover, it shows slow convergence due to the abrupt discontinuities of fields or field derivatives. The FDTD method solves the Maxwell's equations within the unit cell in time domain by applying the periodic boundary condition. In the FDTD method, the eigen-modes are identified as the spectral peaks obtained from the Fourier transform of the field in the time domain. On the other hand, the Finite-Difference Frequency Domain (FDFD) method [8] for analyzing the band diagram of 2-D PCs has been proposed. Authors have demonstrated that the FDFD and/or FDTD method has a high capability for modeling the PC structures without huge computer resources [9]. However, a computation algorithm and the band gap properties have not been investigated when PC includes a loss which is expressed by an electric conductivity $\sigma$. In this paper, a new FDFD scheme for 2D lossy PCs is developed and loss effects for propagation mode are analyzed. It is also shown that the effect of the loss can not be successfully calculated by the FDTD method.

\section{Formulation}

In this section, FDFD algorithm for calculating the 2D PC is briefly summarized and then a new method for lossy material is proposed. Curl equations in frequency domain are given by

$$
\begin{aligned}
\nabla \times \boldsymbol{H} & =j \omega \varepsilon_{0} \varepsilon^{\prime} \boldsymbol{E} \\
\nabla \times \boldsymbol{E} & =-j \omega \mu \boldsymbol{H}
\end{aligned}
$$

where $\varepsilon^{\prime}=\varepsilon_{r}+\frac{\sigma}{j \omega \varepsilon_{0}}$ is a complex relative permittivity, $\mu$ is permeability. Discretizing eqs.(1)(2) using a FDTD cell shown in Fig. 1, the following matrix equation can be obtained

$$
\left[\begin{array}{ccc}
0 & -V_{y} & V_{x} \\
U_{y} & 0 & 0 \\
-U_{x} & 0 & 0
\end{array}\right]\left[\begin{array}{c}
\boldsymbol{E}_{z} \\
\boldsymbol{H}_{x} \\
\boldsymbol{H}_{y}
\end{array}\right]=j \omega\left[\begin{array}{ccc}
\varepsilon_{0} \varepsilon^{\prime} & 0 & 0 \\
0 & -\mu & 0 \\
0 & 0 & -\mu
\end{array}\right]\left[\begin{array}{c}
\boldsymbol{E}_{z} \\
\boldsymbol{H}_{x} \\
\boldsymbol{H}_{y}
\end{array}\right]
$$

where $V_{x}, V_{y}, U_{x}$, and $U_{y}$ are space differential operators for each direction, $\boldsymbol{E}_{z}, \boldsymbol{H}_{x}, \boldsymbol{H}_{y}$ are the field vectors whose elements are given at all edges. 


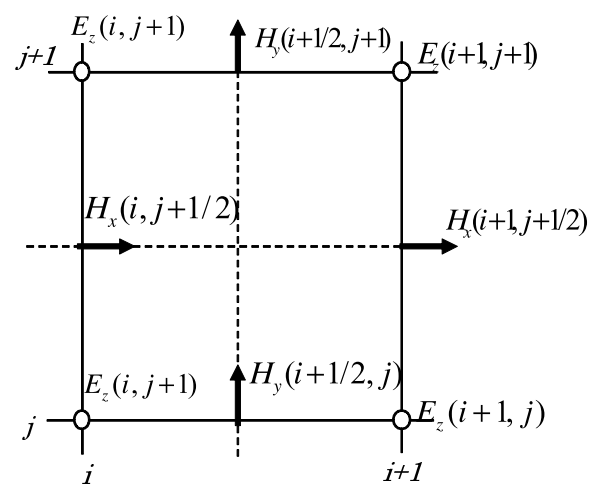

Fig. 1. Yee's mesh for $\mathrm{TM}_{z}$ mode analysis of band structure of $2 \mathrm{D} \mathrm{PC}$

Eq. (3) includes the periodic boundary condition, and should be set in these operators [9]. Eliminating $\boldsymbol{H}_{x}$ and $\boldsymbol{H}_{y}$ from eq. (3), we obtain a matrix equation

$$
\left(-k^{2}+j k \frac{\sigma}{\varepsilon_{r}} Z\right) \boldsymbol{E}_{z}-\frac{1}{\varepsilon_{r}} A \boldsymbol{E}_{z}=0
$$

where $A=\left(V_{y} * U_{y}+V_{x} * U_{x}\right), k^{2}=\omega^{2} \varepsilon_{0} \mu$ and $Z=\sqrt{\frac{\mu}{\varepsilon_{0}}}$. Eq. (4) is the matrix equation for an eigenvlaue $k$, and involves $k^{2}$ and $j k$. Hence normally provided eigenvalue solver cannot be used. However, it can be transformed to a normal equation by adding a trivial equation $\boldsymbol{I} \boldsymbol{E}_{z}=\boldsymbol{E}_{z}$. Then we obtain

$$
\boldsymbol{G} \boldsymbol{X}=k \boldsymbol{X}
$$

where

$$
\boldsymbol{G}=\left[\begin{array}{cc}
j \frac{\sigma}{\varepsilon_{r}} Z & -\frac{1}{\varepsilon_{r}} A \\
\boldsymbol{I} & 0
\end{array}\right], \boldsymbol{X}=\left[\begin{array}{c}
k \boldsymbol{E}_{z} \\
\boldsymbol{E}_{z}
\end{array}\right]
$$

Eq. (5) is the ordinary eigenvalue equation, from which one can find the normalized frequencies

\section{Numerical results}

In order to confirm validity of our method, the band diagram for lossy cylindrical PC is analyzed. Fig. 2 shows the band diagram of a first propagation mode for various electric conductivity values, where $a$ is the size of unit cell and $r$ is the radius of cylinder whose relative permittivity is 8.9. In this calculation we choose $r / a=0.2$. The results calculated by the FDTD are also shown. It is found that the FDTD agrees completely with FDFD for loss less case, however improper values are given by the FDTD calculation when the conductivity is included in the cylinder. This indicates that the FDTD cannot be applied to this range of conductivity values. On the other hand the FDTD agrees again with the FDFD for large conductivity $(\sigma=100)$. The reason is explained as follows. The electromagnetic field in the time domain changes slowly due to the loss in the PC, then the peak of the spectrum tends to shade off. Therefore the detection of the peak search fails. For large conductivity, the peaks of the spectrum are sharpen again because the dielectric $\mathrm{PC}$ tends to a conductor. 


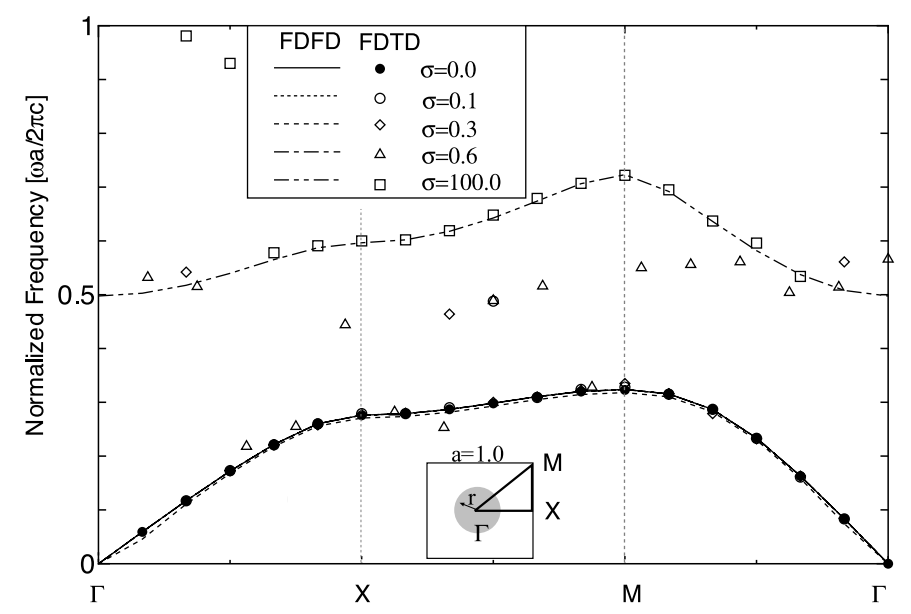

Fig. 2. First propagation mode

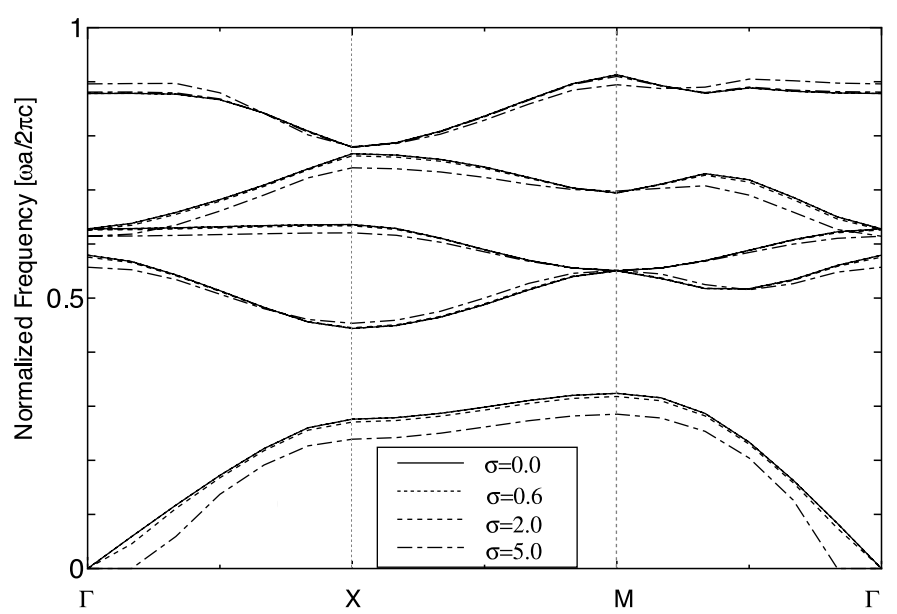

Fig. 3. Band diagram of 2-D PC for lossy dielectric

Fig. 3 show band diagram of first to 5 th propagation modes for different values of electric conductivity. It is found that the proposed FDFD can be calculated properly, and the band gap is rarely affected by loss between $\sigma=0.0$ to 5.0 .

\section{Conclusion}

The FDFD algorithm for precisely calculating of the band diagram of lossy dielectrics has been proposed. This method successfully calculates the band diagram even if conductivity is included in the PC. It has been shown that the FDTD method can not be applied to the range of small conductivity values. 